\title{
Emergency Surgery for Acute Complicated Diverticulitis
}

\author{
Ferdinand Köckerling \\ Department of Surgery, Vivantes Hospital, Berlin, Germany
}

\section{Keywords}

Diverticulitis - Complicated diverticulitis · Hinchey stages · Laparoscopic lavage · Hartmann's procedure

\section{Summary}

Background: The optimal treatment of acute complicated diverticulitis is a matter of debate and has undergone significant changes. Currently, the main focus of surgical treatment concepts is on controlling the emergency situation triggered by acute complicated sigmoid diverticulitis through interventional and minimally invasive measures. Methods: This article presents the current data and recommendations on differentiated treatment of acute complicated sigmoid diverticulitis, which are also summarized in a decision tree. Results: In general, resection of the diverticular sigmoid is needed to treat acute complicated sigmoid diverticulitis, because without resection the recurrence rate is too high at $40 \%$. Since the morbidity and mortality rates associated with emergency resection are extremely high, resulting in the creation of a stoma, efforts are made to control the acute situation through interventional and laparoscopic measures. Therefore, pericolic and pelvic abscesses (Hinchey stages I, II) are eliminated through percutaneous or laparoscopic drainage. Likewise, laparoscopic lavage and drainage are performed for purulent and feculent peritonitis (Hinchey stages III, IV). After elimination of the acute septic situation, interval elective sigmoid resection is conducted. If emergency resection cannot be avoided, it is performed, while taking account of the patient's overall condition, with primary anastomosis and a protective stoma or as discontinuity resection using Hartmann's procedure. Conclusion: Thanks to the progress made in interventional and laparoscopic treatment, differentiated concepts are now used to treat acute complicated sigmoid diverticulitis.

\section{Introduction}

The optimal treatment of acute complicated diverticulitis is a matter of debate and has undergone significant changes with the evolution of surgical techniques and supportive medical care [1]. In the 1980s and 1990s, the majority of cases of complicated diverticulitis were managed by surgical intervention as a means of source control, which traditionally required resection of the perforated segment of colon and creation of an end colostomy [1]. Historically, Hartmann's procedure has been the operation of choice and is still favored by many surgeons [2]. It is associated with morbidity and mortality rates of 43 and 10\%, respectively [2]. Hartmann's procedure also requires a second laparotomy to reverse the stoma and secondary anastomosis of the left colon to the rectum. Approximately $30 \%$ of patients end up with permanent stomas [2]. Colostomy closure after Hartmann's procedure is associated with prolonged hospitalization and a morbidity rate of 33\% [2]. Primary resection and anastomosis with or without stoma is an appealing alternative which may provide acceptable outcomes in terms of morbidity and mortality rate [2].

Currently, the main focus of surgical treatment concepts is on controlling the emergency situation triggered by acute complicated sigmoid diverticulitis through interventional and minimally invasive measures. These include abscess drainage or abdominal lavage and drainage as well as conservative treatment aimed at paving the way for elective sigmoid resection with anastomosis without a stoma. This paradigm shift has been made possible in large part because of advances in antibiotic development, interventional radiology techniques, critical care medicine, and the development of minimally invasive surgery [1].

\section{Definition and Incidence of Acute Complicated Diverticulitis}

The standardized incidence rate of hospitalization for acute diverticulitis was found - on the basis of a sample consisting of 
$20 \%$ of the US population - to have risen from 59 per 100,000 per year to 71 per 100,000 per year from 1998 to 2005 [3]. Overall annual age-adjusted admissions for acute diverticulitis increased from 120,500 in 1998 to 151,900 in 2005 (26\% increase) [3]. Extrapolated to Germany that amounts to around 60,000 cases of inpatient treatment because of acute sigmoid diverticulitis. Acute diverticulitis is divided into uncomplicated and complicated diverticulitis. Acute complicated diverticulitis is mainly stage-divided by the Hinchey classification [4]. The Hinchey classification, developed before the advent of routine computed tomography (CT) imaging, remains the most widely used classification [5] for acute complicated diverticulitis. The original Hinchey classification divided acute complicated diverticulitis into pericolic abscess confined to the mesentery of the colon (stage I), pelvic abscess resulting from a local perforation of a pericolic abscess (stage II), generalized peritonitis resulting from rupture of pericolic/pelvic abscess into the peritoneal cavity (stage III), and feculent peritonitis resulting from the free perforation of a diverticulum (stage IV) [5].

Most patients with acute uncomplicated sigmoid diverticulitis respond to medical treatment and generally experience significant decrease in their abdominal pain, temperature, and white blood cell count within the first $48 \mathrm{~h}$ after initiation of antibiotic treatment [5]. In a minority of patients, non-operative treatment fails and symptoms either persist or worsen. In these cases, urgent or semi-urgent surgery may become necessary during the same hospital stay [5].

In general, percutaneous drainage or surgery is necessary for acute complicated diverticulitis in the Hinchey stages II-IV.

Between 2002 and 2007, data on 1,073,397 patients who were admitted to hospital because of diverticulitis were entered into the American National Inpatient Sample Database. Of these, 78.3\% were emergency admissions, and $21.7 \%$ elective admissions. It was possible to successfully treat $85.92 \%$ of emergency admissions with antibiotics. $1.88 \%$ underwent percutaneous abscess drainage, and surgery was needed for $12.2 \%$ [6]. In other studies, the proportion of patients needing surgical intervention was 25\% [7]. Hence, around $15-25 \%$ of patients admitted to hospital because of acute diverticulitis require urgent percutaneous abscess drainage or surgical treatment.

\section{Diagnosis in Acute Complicated Diverticulitis for Hinchey Staging}

CT imaging has become a standard tool to aid in the diagnosis and Hinchey staging of patients with suspected diverticulitis, to assess disease severity, and to help plan treatment [7]. In a prospective study of 542 patients with acute left-sided diverticulitis, triplecontrast CT scans (intravenous, oral, and rectal) were compared to water-soluble contrast enema. CT scan had a significantly higher diagnostic sensitivity of 0.98 versus $0.92(\mathrm{p}<0.01)$. Only in $29 \%$ of cases with CT-proven abscess did colonic contrast enema show indirect evidence of this [8]. According to the American College of Radiology, CT with intravenous and possibly supplementary oral and rectal contrast is the investigation of choice for suspected acute diverticulitis [9]. The Clinical Practice Guideline Task Force of the American Society of Colon and Rectal Surgeons also recommend CT scans of the abdomen and pelvis as the most appropriate initial imaging modality in the assessment of suspected diverticulitis [7].

\section{Indication for Surgery in Acute Complicated Diverticulitis}

In general, surgery is recommended for complicated diverticulitis after the first episode since the risk of recurrent disease without surgery is very high. However, when age or comorbidities prohibitively increase perioperative risks, it may be appropriate to approach complicated diverticulitis with conservative treatment alone [5].

Patients with multiquadrant peritonitis or overwhelming infection due to purulent (Hinchey stage III) or feculent (Hinchey stage IV) peritonitis are typically acutely ill or appear toxic and require expedited fluid resuscitation, antibiotic administration, and operation without delay [7].

Following successful conservative treatment of mesocolic abscesses (Hinchey stage I) of $<3-4 \mathrm{~cm}$ or mesocolic abscesses (Hinchey stage I) of $>4 \mathrm{~cm}$ or pelvic abscesses (Hinchey stage II) with percutaneous drainage or laparoscopic lavage, elective colectomy should typically be advised, because retrospective data have shown recurrence rates as high as $40 \%$ [7].

\section{Interventional and Surgical Treatment of Acute Complicated Sigmoid Diverticulitis in Hinchey Stages I and II}

Abscess formation, probably the result of a contained perforation, is a common complication of acute diverticulitis and occurs in $15-20 \%$ of patients [7]. The literature supports the concept that percutaneous drainage allows a majority of patients (52-74\%) to avoid urgent operation and undergo interval elective, one-stage colectomy [10-14].

Not all abscesses require interventional drainage for resolution. Abscesses smaller than 3-4 cm in size are often treated conservatively with antibiotic therapy alone [11]. However, patients who do not clinically improve without drainage should undergo percutaneous drain placement or laparoscopic lavage and drainage [7]. Patients with abscesses larger than or equal to $4 \mathrm{~cm}$ can be managed with CT-guided abscess drainage followed by referral for surgical treatment [11].

There are several limitations to percutaneous drainage of diverticular abscesses. First, accessibility is not always possible because of small bowel loops in contiguity with the fluid collection [10]. Second, drainage, when feasible, is not always successful. Reported failure rates for diverticular abscesses range from 15 to $30 \%$. Third, when drainage is feasible and initially successful, abscess recurrence or fistulas may occur, compromising the performance of an elective surgical resection [10]. 


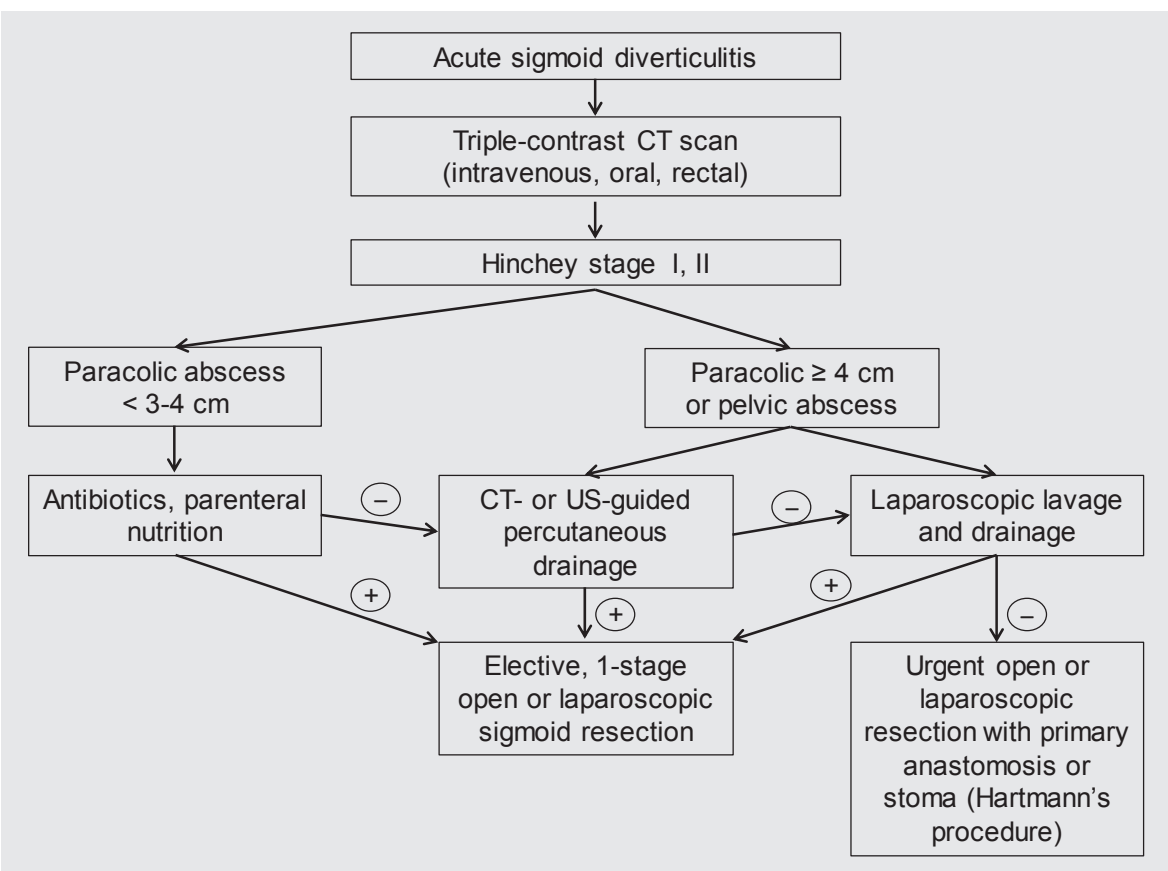
and pelvic abscesses (Hinchey stages I, II) in acute complicated sigmoid diverticulitis.

Patients without an adequate radiographic window to permit safe percutaneous drainage may be candidates for operative drainage that is typically accomplished laparoscopically [7].

In a systematic review [15], it was possible to successfully treat 39 of 44 pelvic abscesses (Hinchey stage II) through laparoscopic lavage and drainage. This has led to the creation of a decision tree for acute complicated sigmoid diverticulitis in Hinchey stages I and II depicted in figure 1.

\section{Open Surgical Treatment of Acute Complicated Sigmoid Diverticulitis in Hinchey Stages III and IV}

In the majority of cases, the first diverticulitis attack is the most dangerous and is associated with the highest perforation risk [16]. Free perforation with generalized purulent or feculent peritonitis often necessitates emergency sigmoid resection, and indeed discontinuity resection of the sigmoid colon using Hartmann's procedure, with its attendant high morbidity and mortality [17]. There continues to be a lively debate about whether sigmoid resection with primary anastomosis, and possibly a protective stoma, or discontinuity resection using Hartmann's procedure should be performed in cases of free perforation with purulent (Hinchey stage III) or feculent (Hinchey stage IV) peritonitis. 16 retrospective studies have been published on this topic and their results analyzed in 3 systematic reviews [18]. The systematic reviews [2, 17, 19] showed that discontinuity resection using Hartmann's procedure was associated with a mortality rate of $15-19 \%$, whereas for primary anastomosis it was only $4.9-9.9 \%$. Only for $45 \%$ of patients a reversal of Hartmann's procedure was performed, i.e. continuity was later restored. Besides, the reversal operation was associated with a morbidity rate of $30-40 \%$ and a mortality rate of $5 \%$. Furthermore, the quality of life following discontinuity resection and subsequent reversal operation was poorer than after primary anastomosis [20].

In addition to the systematic reviews, 2 prospective randomized multicenter trials have been published in the past 2 years $[21,22]$. The first study did not find any difference in morbidity and mortality between primary anastomosis with a protective ileostomy and Hartmann's discontinuity resection in similar patient collectives. Reversal ileostomy was carried out significantly more often than reversal of Hartmann's procedure ( 90 vs. $57 \%$; $p=0.005$ ). Reversal ileostomy had a lower complication rate, shorter duration of operation, shorter hospital stay, as well as lower costs [21]. Likewise, the second study did not discern any difference in morbidity and mortality between primary anastomosis with a protective ileostomy and Hartmann's discontinuity resection. However, in that study the stoma reversal rates were identical (64.3 vs. $60 \%$; $\mathrm{p}=$ 0.659) [22].

Based on the available data, it is no doubt advisable to use the patient's risk profile as the chief determinant when deciding whether to opt for primary anastomosis with a protective stoma or discontinuity resection using Hartmann's procedure. If the patient has comorbidities (ASA III or IV), severe sepsis, or long-standing feculent peritonitis, preference should definitely be given to Hartmann's discontinuity resection. If the patient is in a relatively good general state and there is peritonitis of recent onset and only a mild septic reaction, creation of a primary anastomosis, possibly with a protective stoma, can be considered [23].

\section{Laparoscopic Treatment of Acute Complicated Sigmoid Diverticulitis in Hinchey Stages III and IV}

In recent years, laparoscopic treatment of perforated sigmoid diverticulitis has been increasingly used as an alternative to open 
surgery for Hinchey stages III and IV [15, 24]. Sigmoid resection is now completely omitted in emergency procedures, and only abdominal lavage and drainage are performed. A systematic review reported the findings for 231 patients treated by means of laparoscopic lavage and drainage. Here 44 patients had Hinchey stage II, i.e. abscess of the small pelvis, 178 patients had Hinchey stage III with purulent peritonitis, and 8 patients had Hinchey stage IV with feculent peritonitis [15]. It was possible to successfully treat $95.7 \%$ of patients using lavage and drainage alone. The morbidity rate was only $10.4 \%$, and mortality was $1.7 \%$. It was possible to conduct elective sigmoid resection in the later course for $87 \%$ of patients. These positive outcomes have since been confirmed in 17 retrospective studies with a total of 768 patients [18].
Two prospective randomized multicenter trials are currently being carried out, one in the Netherlands [25] and one in Scandinavia [26], with the aim to ascertain the role of laparoscopic lavage and drainage compared with resection of purulent and feculent perforated sigmoid diverticulitis (Hinchey stages III, IV). The indication for laparoscopic lavage and drainage should be based on stringent criteria until such time as the findings of these studies are published. Feculent peritonitis with a visible perforation site no doubt constitutes an exclusion criterion [18].

\section{Disclosure Statement}

The author has no conflict of interest or financial ties to disclose.

\section{References}

1 Dharmarajan S, Hunt SR, Birnbaum EH, Fleshman JW, Mutch MG: The efficacy of nonoperative management of acute complicated diverticulitis. Dis Colon Rectum 2011;54:663-671.

2 Abbas S: Resection and primary anastomosis in acute complicated diverticulitis, a systematic review of the literature. Int J Colorectal Dis 2007;22:351-357.

3 Etzioni DA, Mack TM, Beart RW, Kaiser AM: Diverticulitis in the United States: 1998-2005. Ann Surg 2009;249:210-217.

4 Andersen JC, Bundgaard L, Elbrønd H, Laurberg S, Walker LR, Støvring J: Danish national guidelines for treatment of diverticular disease. Dan Med J 2012;59: C4453.

5 Stocchi L: Current indications and role of surgery in the management of sigmoid diverticulitis. World J Gastroenterol 2010;16:804-817.

6 Masoomi H, Buchberg BS, Magno C, Mills SD, Stamos MJ: Trends in diverticulitis management in the United States from 2002 to 2007. Arch Surg 2011;146:400-406.

7 Feingold D, Steele SR, Lee S, Kaiser A, Boushey R, Buie WD, Rafferty JF: Practice parameters for the treatment of sigmoid diverticulitis. Dis Colon Rectum 2014;57: 284-294.

8 Ambrosetti P: Acute diverticulitis of the left colon: value of the initial CT and timing of elective colectomy. J Gastrointest Surg 2008;12:1318-1320.

9 American College of Radiology: ACR Appropriateness Criteria $^{\circledR}$ : Left Lower Quadrant Pain - Suspected Diverticulitis. ACR, 2014. www.acr.org.

10 Durmishi Y, Gervaz P, Brandt D, Bucher P, Platon A, Morel P, Poletti PA: Results from percutaneous drainage of Hinchey stage II diverticulitis guided by computed tomography scan. Surg Endosc 2006;20:1129-1133.

11 Siewert B, Tye G, Kruskal J, Sosna J, Opelka F: Impact of CT-guided drainage in the treatment of diverticular abscesses: size matters. AJR Am J Roentgenol 2006; 186:680-686.
12 Brandt D, Gervaz P, Durmishi Y, Platon A, Morel P, Poletti PA: Percutaneous CT scan-guided drainage vs antibiotherapy alone for Hinchey II diverticulitis: a casecontrol study. Dis Colon Rectum 2006;49:1533-1538.

13 Ambrosetti P, Chautems R, Soravia C, Peiris-Waser N, Terrier F: Long-term outcome of mesocolic and pelvic diverticular abscesses of the left colon: a prospective study of 73 cases. Dis Colon Rectum 2005;48:787-791.

14 Kaiser, AM, Jiang JK, Lake JP, Ault G, Artinyan A, Gonzalez-Ruiz C, Essani R, Beart RW: The management of complicated diverticulitis and the role of computed tomography. Am J Gastroenterol 2005;100:910-917.

15 Toorenvliet BR, Swank H, Schoones JW, Hamming JF, Bemelman WA: Laparoscopic peritoneal lavage for perforated colonic diverticulitis: a systematic review. Colorectal Dis 2010;12:862-867.

16 Anaya DA, Flum DR: Risk of emergency colectomy and colostomy in patients with diverticular disease. Arch Surg 2005;140:681-685.

17 Constantinides VA, Tekkis PP, Athanasiou T, Aziz O, Purkayastha S, Remzi FH, Fazio VW, Aydin N, Darzi A, Senapati A: Primary resection with anastomosis vs Hartmann's procedure in nonelective surgery for acute colonic diverticulitis: a systematic review. Dis Colon Rectum 2006;49:966-981.

18 Holmer C, Kreis ME: Diverticular disease - choice of surgical procedure. Chirurg 2014;85:308-313.

19 Salem L, Flum DR: Primary anastomosis or Hartmann's procedure for patients with diverticular peritonitis? A systematic review. Dis Colon Rectum 2004;47: 1953-1964.

20 Vermeulen J, Gosselink MP, Busschbach JJV, Lange JF: Avoiding or reversing Hartmann's procedure provides improved quality of life after perforated diverticulitis. J Gastrointest Surg 2010;14:651-657.
21 Oberkofler CE, Rickenbacher A, Raptis DA, Lehmann K, Villiger P, Buchli C, Grieder F, Gelpke H, Decurtins M, Tempia-Caliera AA, Demartines N, Hahnloser D, Clavien PA, Breitenstein S: A multicenter randomized clinical trial of primary anastomosis or Hartmann's procedure for perforated left colonic diverticulitis with purulent or fecal peritonitis. Ann Surg 2012;256:819-827.

22 Binda GA, Karas JR, Serventi A, Sokmen S, Amato A, Hydo L, Bergamaschi R: Primary anastomosis vs nonrestorative resection for perforated diverticulitis with peritonitis: a prematurely terminated. Colorectal Dis 2012;14:1403-1410.

23 Constantinides VA, Heriot A, Remzi F, Darzi A, Senapati A, Fazio VW, Tekkis PP: Operative strategies for diverticular peritonitis - a decision analysis between primary resection and anastomosis versus Hartmann's procedures. Ann Surg 2007;245:94-103.

24 Myers E, Hurley M, O’Sullivan GC, Kavanagh D, Wilson I, Winter DC: Laparoscopic peritoneal lavage for generalized peritonitis due to perforated diverticulitis. Br J Surg 2008;95:97-101.

25 Swank HA, Vermeulen J, Lange JF, et al.; Dutch Diverticular Disease (3D) Collaborative Study Group: The ladies trial: laparoscopic peritoneal lavage or resection for purulent peritonitis and Hartmann's procedure or resection with primary anastomosis for purulent or faecal peritonitis in perforated diverticulitis (NTR2037). BMC Surg 2010;10:29.

26 Thornell A, Angenete E, Gonzales E, Heath J, Jess P, Läckberg Z, Ovesen H, Rosenberg J, Skullman S, Haglind E: Treatment of acute diverticulitis laparoscopic lavage vs resection (DILALA): study protocol for a randomized controlled trial. Trials 2011;12:186. 